\title{
Potential application of autonomous and semi- autonomous robots in the study of animal behaviour
}

\author{
Á. Miklósi*, L. Gerencsér** \\ *MTA-ELTE Comparative Ethology Research Group, Eötvös University Budapest, Hungary \\ Department of Ethology, Eötvös University, Budapest, Hungary \\ *amiklosi62@gmail.com, lindus@yahoo.com
}

\begin{abstract}
In this review we aim to facilitate closer contact between animal behaviour and robotics. In recent years cognitive psychology and cognitive ethology became interested in the mental mechanisms controlling the animal mind. However, this theoretical interest is hampered by several methodological problems. Interactive robots can offer a novel way to investigate cognitive skills in animals. It may be useful to construct robots that are specifically designed to interact with animals.
\end{abstract}

\section{INTRODUCTION}

There have been two main driving forces for studying animal behaviour by the means of scientific tools. First, the field on comparative psychology (e.g. [1]) emerged at the end of the $19^{\text {th }}$ century, in which animals were used instead of humans to investigate basic questions on the mechanisms of learning. At that time the flexible and enormously powerful learning abilities of humans were seen as a specific feature discriminating man from animals. Somewhat paradoxically, however, since humans turned out to be too complex and tiresome for laboratory investigations, researchers focused on a few animals that could be kept in the laboratory (e.g. rats, Rhesus monkeys, dogs, cats [2]). It was just unfortunate that the success with describing the phenomena of learning in terms of stimulus-response patterns (e.g. 'laws of effect' [2]) decreased interest in rich mental processes because the majority of scientists of that time e.g. [3] believed (and some still believe) that robust associative behaviour models have the power to explain most if not all mental processes in animals. Furthermore such investigations on learning are constrained to a very limited experimental setting (e.g. Skinner box, in which the rat learns to push a treadle with its paw) in which the action of the animal becomes only an indirect marker for the mental events (for further discussion see [4]).

Researchers like Konrad Lorenz, Niko Tinbergen (and many others) who had training as zoologists had a quite different aim when they got interested in the study of animal behaviour. Their most important insight was that, generally speaking, (substantial part of) the behaviours of an animal species (e.g. eating habits, courtship behaviour etc.) were as typical features as its morphological peculiarities. However, they soon realised that the study of behaviour was methodologically quite different from the study of morphology, so the discipline called ethology emerged ('ethos' - habit/character + 'logos' science). The close study of species specific behaviours like courtship or aggression naturally led to the issue of how to deal with the problem of instinct e.g. [5]. One possible solution was describe this mental phenomenon in a species specific dichotomy of the 'sign stimulus' and the 'fixed action pattern'. The former notion indentified a simple or complex part of the environment that controlled the emergence of the behaviour, while the later was characterised as being dependent on the former, and relatively free from other environmental influences.

Although in general the concepts of early comparative psychologists and early ethologists shared many common features (both neglected to study the role of the 'mind' in behaviour, and stressed the role of environmental stimuli), they got into heavy debate on the significance of individual experience ('learning') in the emergence of behaviour. Supported by their observations in the laboratory, comparative psychologists argued that behaviour emerges as an individual response to environmental challenges. On the contrary, ethologists (working in an evolutionary framework) underlined the role of genetic factors [6].

Although the waves of the debate are today considerably smaller, and both parties mutually agree that both inherited and environmental factors play a role in the emergence of most behaviours, this reconciliation in itself had not brought us much closer in the understanding of behaviour control in general.

\section{COGNITIVE APPROACH TO BEHAVIOUR}

In the psychological literature the 'rediscovery' of the human mind in the 1950s is often referred to as a 'revolution', and this was the time for the birth of cognitive psychology. Cognitive psychologists understand the mind as a dynamic 'information processing system' in terms of perception, memory, decision making, and problem solving ability. Although the idea of the mind as an information processing system shows some analogies with the computer which became a radically new device in those times, in cognitive psychology the mind is rather interpreted in terms of inner 'mental states' like beliefs, intentions, desires etc. which does not fit well with the computer metaphor.

The question on the nature of animal mind was raised within the discipline of ethology by the famous American zoologist Donald Griffin. The title of his book 'The question of animal awareness' [7] was a real provocation in the face of hard-core ethologists who never thought (or dared to think) that the subjects of their behavioural observations may also be controlled by similar mental processes to that of humans. With cognitive psychology becoming slowly interested in the comparative and evolutionary studies of apes and monkeys, some researchers entertained the idea that mental representation 
of the other's mental state (mental state attribution) may be advantageous for competition in animal groups. Gaining advantages by foretelling the other's next move by the means of attributing some knowledge, belief etc to the companion ('Machiavellian intelligence') became a central idea for explaining the evolution of complex social cognitive abilities in apes and humans [8].

Cognitive ethology is aiming to follow the Darwinian tradition to provide an evolutionary and comparative study of the animal mind. However, despite strong arguments by Alan and Bekoff [9] in favour of cognitive ethology, the lack of a widely acknowledged methodology convinced main stream researchers that this field is infected with subjectivity and aims to study elusive mental phenomena [1]. Many, including the present authors, still believe that cognitive ethology has the potential to provide a unique perspective on the animal mind but future research should make goals and means more explicit (see below).

III. PROBLEMS WITH THE COGNITIVE APPROACH: ARE THERE BEHAVIOURAL MARKERS FOR 'COGNITIVE STATES'?

Right from the beginning the results of cognitive ethologists were looked at with various levels of scepticism. One of the most serious critics was Cecilia Heyes [10] who was very accurate to point out methodological problems with the research. She did not have a hard time because cognitive ethologists put the stakes quite high: they wanted to show that apes are able to attribute mental states to humans or conspecific companions. Based on the formal logic of 'associationism' Heyes could efficiently dismantle the cognitive interpretations, and provide alternative explanations which reduce the phenomena to associative processes. Importantly, many associative mental models have the power to work in simple computational models but there is no proof that all mental phenomena in animals can be reduced to an explanation based on associative processes. Byrne and Bates [4] and many others argue that such models are applied only 'post hoc' as explanations and this does not imply that they are functionally equivalent with the studied phenomenon.

From the ethologists' point of view the problem is whether cognitive states can be characterised by specific behavioural markers which could exclude alternative explanations. In other words is there an act or some specific combination of acts that stands for mental state 'X' (e.g. 'seeing'). Such behavioural markers ('I see you') are easily generated in systems that use language because a word can easily become such a label. Actually, the problems of interpreting the mind of the preverbal infant are comparable to the inquiry about animal mind. One quite widely applied approach in infants relies on looking time change as a marker for certain mental processes (violation of expectation paradigm) [11]. It is assumed that infants look longer if they see impossible or unexpected events. Researchers using this method claim that concepts like object permanence or solidity emerge very early in the human infants, but there is an ongoing debate about the validity of the basic assumption of the paradigm.

It may be the case that animals or human infants allow simply too few input-output states for researchers to find out precisely the underlying structure of the mind. In other words, our way of measuring these input-output relationships is too crude for providing a good estimation about the necessary complexity for explaining the mind.

\section{TWO 'CLASSIC' EXAMPLES FOR THE INTERPRETABILITY PROBLEM}

In line with the above notion the study of behaviour does not provide a straight forward interpretation of the mind in terms of cognitive states. Simple mental states like 'intentionality', which are quite trivial in case of adult humans, are a nightmare for those who want a waterproof evidence for animals. Although, one can put all these efforts aside and accept that animal behaviour at a face value resembles human behaviour in terms of goal directedness (which is a way of interpreting intentionality) but this fails to uncover underlying mental mechanisms that in principle could be quite different.

As mentioned above one way of circumventing the problem is to look for behavioural indices of mental states. For example, in the case of intentional communication researchers studying preverbal human infants converge on a list of such behavioural markers (criteria) that 'define' the presence of an intentional act (summarized in [12]). These include social use of signals, manipulation of attention, gaze alternation, persistence in and elaboration of communicative behaviour. Interestingly, this list was recovered from several researchers who used only a few criteria, however, it is still questionable whether (1) these criteria can be used in cross-species comparison, (2) whether some criteria are more important than others, (3) whether the lack of one or more criteria excludes intentionality, and whether (4) the criteria may vary from behaviour to behaviour.

Research on social learning is particularly interested in describing mental mechanisms by the means of which the observer is able to utilise third person information for his own advantage. If a chicken observes another chicken using its leg to push a treadle for obtaining food, it will also use this action if it finds itself in the same situation. After many years of work most researchers seem to agree on four broad categories (mechanisms) of social learning: enhancement, observational conditioning, emulation and imitation [13]. It is not our intention here to elaborate these categories of learning, but to stress that such strict separation makes real (natural) situations very difficult to interpret. For example, how can we interpret if a dog increases its effectiveness of detouring a fence after observing an object (e.g. bucket) making a detour around one end of the fence? Does learning from an object (a box) fulfil conditions of 'social learning'? Is the dog learning about the path, the corner or that 'corners can be circumnavigated' during the observation?

Somehow animal (and human) behaviour seems to be much richer than expected and linguistic labels seem to be providing constrains for the scientific research on mental processes. While our everyday words for mental phenomena may serve well in interpreting our own or others' behaviour objectively (He wants the banana.) or subjectively (I feel he wants the banana.), but they fail to be useful for being the building blocks of mental models. Defining and providing an existence for a linguistic label (e.g. 'intention') in the mind of animals seems to be an effort in vain. Although research may still advance using this paradigm, the time may have come to look for alternative ways of investigation. The use of artificial agents, collectively referred to as robots, may offer some 
new avenues for future research that aims at studying the mind from an ethological perspective.

\section{ETHOLOGY AND ROBOTICS: DIVERGENT HISTORY, CONVERGING FUTURE}

Ethological analysis of animal behaviour always relies on a functional analysis. This includes the description of the particular niche that is inhabited by the species, the estimation of the possible challenges of living and the behavioural means that support the species' survival. Thus the key question for ethologists is how successfully the species manages to live under certain constrains and conditions.

A further important insight is that evolutionary interpretations implicate that animal behaviour is the result of a process of adaptation. Adaptation does not make behaviour perfect, indeed if looked at in isolation most behaviour systems underperform. Thus adaptation can be seen as a process which drives the animals towards a local optimum given many trade-offs in their niche. Recent insights in behavioural ecology also suggest that the local optimum represents the mean value, and individuals in a population may deviate considerably from this point. In evolving systems this also allows for relatively rapid changes in this optimal mean by altering the ratio of individuals with different character within a population.

It is quite unfortunate that such analysis is often missing from robotics, and the possible role of the environment is not taken seriously. The emergence of robots, at least in a general sense of the word, was possible after engineers realised that complex working processes (e.g. car making) can be divided into small, repeatedly performed actions if they can provide a constant environment for executing these actions. Although contemporary industry robots perform very complex tasks with great precision, this is only possible because they do not have to bother with any change in the environment (because there is no change - at least from their perspective).

Recently engineers have become interested in the problem of whether some robots could function in 'real' environments. It retrospect it seems that they did not take the emerging issues seriously, that is, what it really takes to move a system from a invariant environment to a variable one, in which the recognition and prediction of the events becomes a crucial issue. Moreover roboticists suggested or at least did not deny that some types of robots may be able to share the niche of humans which is one of the most complex ones on Earth. This paradox is nicely supported by a 'youtube' video that shows a female android trying to mimic a human smiling gesture but the viewer can see that the android moves on a wheeled platform, and its whole body is lurching back and forward giving the impression of falling over in any minute.

In a famous book on evolution Eörs Szathmáry and John Maynard Smith [14] identified five critical steps in evolving systems. Humans have passed all evolutionary levels in a sequential order. However, it seems that robot building starts at the last step of this process as if it avoided the ones at lower level. This case is analogous to a situation when a novice interested in mathematics wants to start with understanding differential equation. In other words, robotics should strive for being more realistic. Even the word 'autonomy' is used in a very different meaning in evolutionary biology and robotics, and especially in the latter autonomy is used also very loosely, that is, in all cases autonomy is restricted to a narrow range of functions and does not apply to the robot as a whole.

Importantly this somewhat pessimistic view does not suggest that robots have no future! On the contrary, present day's engineers together with other scientists should work on elaborating realistic targets for robots of our time.

\section{ANIMALS AS TESTBEDS FOR INTERACTIVE ROBOTS}

Earlier we have implicated that robotics, especially social robotics [15] could profit from the study of animal behaviour [16]. More particularly, we have argued that robots designed for social interaction with humans could be using behavioural patterns which are derived from social animals, particularly those which have been sharing the life of humans (e.g. dogs). In a series of experiments we have provided a methodology how detailed observation of human-dog interaction can lead to insights for developing more believable social behaviour for robots [17].

If such robots could be made functional then this approach may offer a new possibility for using robots in animal-robot interaction. One key question in ethological research involves the investigation of environmental stimuli (events) that affect and guide behaviour. This research agenda was very successful in identifying sign stimuli which have an innate basis, control complex species specific behaviour, and guide further learning about the environment.

A recent review [18] provided a detailed state of art of animal-robot interaction which seems to be an emerging new field in the study of animal behaviour. The authors listed several ways how robots can be used to investigate animal behaviour in a novel way. For example, they can replace the handmade replicas of experimental stimuli, making these stimuli more lifelike and capable to interact with the living organism. Several such experiments were carried out with groups of cockroaches and various fish species. In these cases the robot was regarded by the animals as a conspecific, and in this way allowing scientists to manipulate the movement of the group in space. In principle, robots could also participate in more complex social interactions with animals in different situations.

It is important to note that most of these robots are remote controlled either by an assistant directly or by a program that determines its behaviour step by step. In other words such systems are rarely 'reactive', partly because their perceptual abilities are limited to assess the action of the animal partner. Thus human assistants may provide a simple and cheap option to control these robots. Nevertheless, roboticists may consider animal-robot interaction as a possible scenario for developing their robots, and making them more autonomous. Instead of using the human as a partner with very complex perceptual, behavioural and cognitive skills, animals may provide a more realistic target for developing interactive robots. In this case robots will not be reduced to a 'replica' of some living being assisting the ethologists in 
their research but the aim would be to make these robots as independent ('autonomous') as possible in their interactions with animals. We believe that the community of roboticists should take this opportunity more seriously and in addition to helping ethologists in their work, they should be a driving force for designing such robots which could be on par with animals in certain forms of interaction. We claim that the experience gained during the design of interactive robots for animals will raise problems and ideas which eventually help to develop more believable robots for humans.

\section{ACKNOWLEDGMENT}

The present research (Swarmix) is gaining support from the Swiss National Science Foundation. A.M. is a member of the ELTE-MTA Comparative Ethology Research Group (01 031). Some ideas presented in this paper developed during an earlier project (LIREC EU FP7 ICT-215554) supported by the European Union and an ongoing project of ESF Research Networking Programme CompCog. Support of ETOCOM project is also acknowledged (TAMMP-4.2.2-08/1/KMR-20080007).

\section{REFERENCES}

[1] S.J. Shettleworth"Clever animals and killjoy explanations in comparative psychology". Trends in Cognitive Sciences, 14, 477-481, 2010.

[2] E. L. Thorndike "Animal intelligence: experimental studies". New York: Macmillan, 1911.

[3] B.F. Skinner'The Behavior of Organisms". New York: AppletonCentury-Crofts, 1938.

[4] R.W. Byrne \& L.A. Bates "Sociality, evolution \& cognition" Current Biology, 17, R714-723, 2007.

[5] N. Tinbergen"The Study of Instinct". Oxford, Clarendon Press, 1951.

[6] S. D. Lehrman "A Critique of Konrad Lorenz's Theory of Instinctive Behavior". The Quarterly Review of Biology, 28, 337-363, 1953.

[7] D. Griffin, "Question of Animal Awareness". Rockefeller University Press. 1976.

[8] R.W. Byrne \& A. Whiten" Machiavellian Intelligence: Social Expertise and the Evolution of Intellect in Monkeys, Apes, and Humans". Oxford University Press. 1985.

[9] C. Allen \& M. Bekoff "Species of mind: The philosophy and biology of cognitive ethology". MIT Press, 1997.

[10] C. Heyes, C. "Theory of mind in nonhuman primates". Behaviour and Brain Sciences, 21, 101-114, 1998.

[11] R. Baillargeon, "Representing the existence and the location of hidden objects: Object permanence in 6- and 8-month-old infants. Cognition, 23, 21-41, 1986

[12] D. Leavens, J.L. Russell, \& W.D. Hopkins, "Intentionality as measured in the persistence and elaboration of communication by chimpanzees (Pan troglodyes)". Child Development, 76, 291306, 2005.

[13] T.R. Zentall, "Imitation and other forms of social learning in animals: evidence, function, and mechanisms". Cybernetics and Systems, 32, 53-96, 2001.

[14] J. Maynard Smith, \& E. Szathmáry, "The Major Transitions in Evolution". Freeman \& Co., Oxford. 1995

[15] T. Fong, I. Nourbakhsh, K. Dautenhahn "A survey of socially interactive robots. Robotics and Autonomous Systems 42, 143166, 2003.

[16] Á. Miklósi, M. Gácsi,”On the utilization of social animals as a model for social robotics". Frontiers in Psychology, 3, 75. 2012.

[17] A. Kis A. M. Gácsi, B. Turcsán, Á.Miklósi, Á “A plausible way for optimizing human-robot interactions: evaluating the interactions of human-dog dyads". Interaction Studies (in press).
[18] J. Krause, A.F.T. Winfield \& J-L. Deneubourg "Interactive robots in experimental biology". Trends in Ecology and Evolution, 26, 369-375, 2011. 